\title{
Maximizing Efficiency Of multiple-Path Source Routing in Presence of Jammer
}

\author{
Kande Archana ${ }^{1}$, Linugtla Laxmi ${ }^{2}$, Dr.A.Govardhan ${ }^{3}$, \\ ${ }^{I}$ Asst.Professor, (Department of CSE, MLR Institute of Technology, Hyderabad, India) \\ ${ }^{2}$ Asst.Professor (Department of CSE, MLR Institute of Technology, Hyderabad, India) \\ ${ }^{3}$ Professor (Department of CSE \& DE (Director of Evaluation) of JNTU Hyderabad, India)
}

\begin{abstract}
Routing traffic through multiple paths is known as multipath routing. There was considerable research in the area of multiple-path source routing to overcome the problem of jamming. Many jammingaware approaches existed. This paper explores the jamming aware concept of [1] with empirical studies using NS2. Moreover it also explores the possibility of relaxing the assumption of [1] with respect to in-network inference of correlations among related variables. In this implementation the source node allocates traffic to the available paths based on the awareness of jamming details at nodes. The practical implementation makes use of portfolio selection as explored in [1] and tries to explore the in-network inference of correlations among estimated random variables. The algorithm used to achieve this ensures that all available paths are optimally utilized without congestion while maximizing throughput. The simulation results revealed that the network is capable of performing jamming aware allocation of traffic.
\end{abstract}

Index Terms-Multiple path source routing, traffic allocation, jamming, optimization, and in-network inference

\section{Introduction}

Jamming in various kinds of networks can disrupt the normal flow of data thus causing problems to the network and its users. It also causes various kinds of attacks such as DoS (Denial of Service) attack [2]. As jamming is done at physical layer it can have its consequences. Therefore physical layer solutions can overcome this kind ofproblem. The solutions such as beamforming and spread-spectrum come under the category of physical layer solutions. These solutions force jammers to utilize more resources in order to achieve their goal of attacking network thus discouraging such attacks. However, there were incidents in the recent path where the jammers were able to use cross-layer protocol information also for making jamming attack thus they were able to reduce the consumption of resources required for an attack. This resource utilization was reduced to a greater extent when they use cross layer protocol information making the attacks feasible. Mainly adversaries focused on the MAC implementations and link layer[3] [4]. They also targeted correction protocols and link layer error detection mechanisms [5]. This necessitated the research in order to find new methods for anti-jamming to be incorporated into higher layer protocols such as channel surfing [6]. Another such solution could be the process of routing the traffic around the jammed areas.

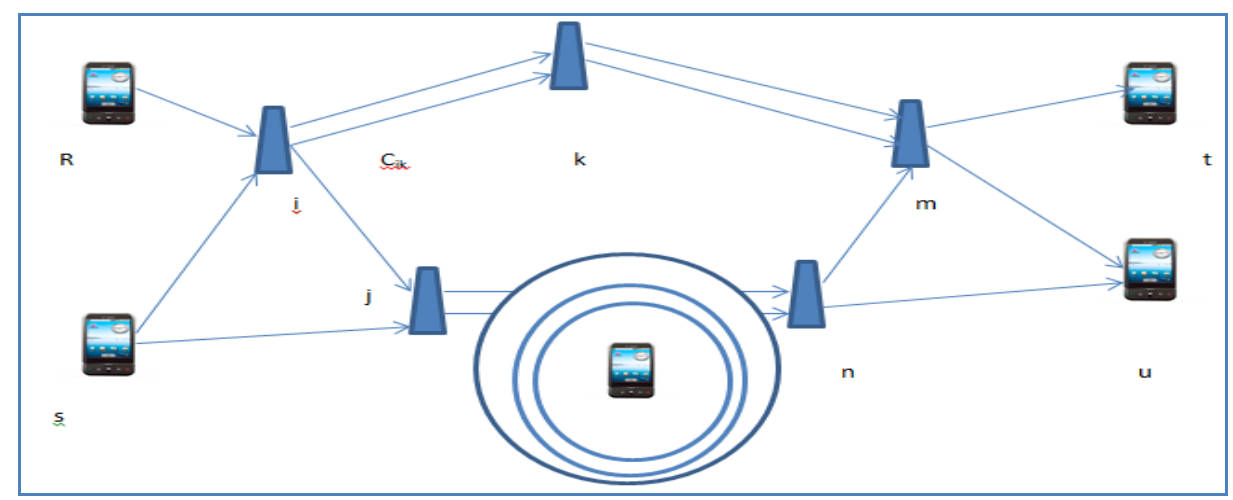

Fig. 1-Shows the presence of jammer in the network

There is considerable diversity in the available anti-jamming methods. The diversity of techniques includesmultiple routing paths, different MAC channels, and multiple frequency bands. This paper makes use of multiple routing paths to find solution for jamming. The multiple path source routing protocols include MPDSR protocol [7] AODV (Ad-Hoc On-Demand Distance Vector protocol, and DSR (Dynamic Source Routing). In all these protocols the source node sends data through multiple available paths. For this reason the source node must be intelligent to know the traffic allocation which does not cause jamming. The consideration of effect of 
jamming is important. As explored in [1], it is essential to know the previous jamming statistics before taking routing decisions besides using portfolio selection approach. This will increase the throughput in the network while avoiding jamming problems. It is essential for the source node to be able to estimate the effect of jamming on throughput. Each source node in the network must be aware of jamming statistics and thus take multiple path source routing decisions effectively. However, the jamming awareness depends on many factors and unknown parameters as adversaries use different strategies for jamming and also location of jammers. Therefore the finding the impact of jamming on network throughput is probabilistic in nature. In order to simulate the dynamic nature of jamming attack, we used the model provided in [1] for packet error rate to be incorporated at each node which considers the mobility and dynamics of jamming. As jamming effect at individual nodes is probabilistic, the throughput achieved by source and destination nodes is also non-deterministic. Therefore this paper the stochastic approach followed in [1]. The practical implementation of multiple-path source routing of this paper contributes the following.

- We formulate the process of jamming-aware traffic allocation in a network in the presence of jamming as lossy network flow optimization problem.

- We also consider portfolio theory for best allocation of traffic [8].

- An algorithm is used for network utility maximization as explored in [1].

- We implemented methods in NS2 in order to characterize jamming impact locally and take jammingaware traffic allocation decisions.

- We also explore the in-network inference of correlation among the related variables. Actually this was based on an assumption in [1].

The rest of the paper is organized into some sections. Section II reviews literature pertaining to jamming aware traffic allocation and anti-jamming techniques. Section III provides our approach used for jamming aware traffic allocation. Section IV provides experimental results and evaluation. Section V provides conclusions.

\section{Related Work}

This section briefs about the prior work done on anti jamming and jamming aware traffic allocation. The effects of jamming attacks were explored in [9]. The jamming can have its effects on the network and its users of the network. The denial of service attacks possibilities when traffic is jammed is explored in [2] with respect to end to end communication.Jammers used different techniques and they keep changing over a period of time the approach they follow for jamming making it difficult to develop a perfect anti-jamming technique. The possibility of attackers making use of certain MAC implementations and link layer are described in [7] and [10]. The usage of error correction protocols and the link layer error detection for anti-jamming is explored in [5]. In order to overcome this problem researchers started investigating the ways and means to incorporate the defensive mechanisms against jamming attacks into the higher-layer protocols and the ways to route around the jammers [10], [12]. Research has been around on jamming attacks and also the use of multiple path source routing protocols such as DSR, AODV and MPDSR [11][7]. All these protocols are capable of allowing a network source node to send data to destination node through multiple available paths. In [1] jamming aware traffic allocation is explored along with portfolio selection theory in order to allocate traffic for more benefits in terms of maximizing throughput in the presence of jammers. The portfolio selection theories are in [8], AND [12]. This theory is related to finance domain where it talks about funds investment. In investor, before investing funds, thinks about various possibilities or portfolios that tells him the possible outcomes in each portfolio thus helping his taking good investment decisions. This is used in [1] to explore jamming aware traffic allocation effectively. In addition to this, this paper also relaxes assumption pertaining to the in-network inference of correlation among various variables.

\section{FINDING THE IMPACT OF JAMMING} System Model and Characterization

A wireless network is considered for making experiments on jamming aware traffic allocation where a source node can send packets to destinantion through multiple paths with awareness of jamming. We assume that the communication is unicast model. Another assumption is that the source node $\mathrm{S}$ has no prior knowledge about the jammer's goals and the locations and number of jammers is unknown. The communication between the source node and destination node using multiple-path source routing in presence of jammers is illustrated in fig. 2 . 


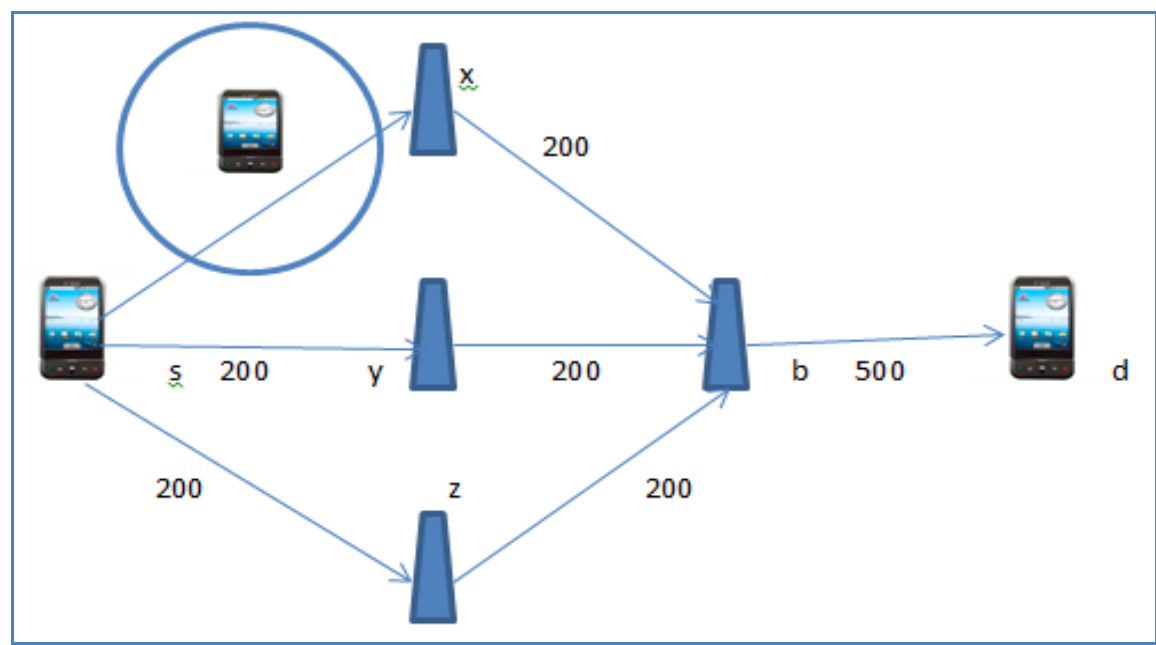

Fig. 1 -Source and destination with multiple paths

As seen in fig. 2, six nodes exist in the network where the source node and destination nodes are labeled "s" and "d" respectively. On each edge the numbers indicate the link capacities. The value represents number of packets. This paper assumes that the source node sends packets to destination at the rate 300 when there is no presence of jammers in the network. Traffic allocation is made equally on all the available paths. The source node which is supposed to senddata to destination node is aware of jamming statistics on various paths and also in-network inference correlation among variables. Based on the jamming statistics, the decisions are made by the source node while allocating traffic to multiple paths.

Through analysis and statistics pertaining to jamming, the estimation of success rate of packets is done prior allocating traffic. There are many considerations such as the presence of jammer, path loss behavior of wireless medium, signal power of jammer, the distance between the jammer and source node, the in-network inference correlations etc. As a matter of fact, the source node is not aware of the location of jammers. Simple usage of an analytical model may not be practical. For this reason we consider, the packet success rate as a random process which is dynamic in nature. Each node, before sending data to destination, is supposed to estimate the packet success rate, in-network inference statistics, various parameterin order to correctly characterize the uncertainity and process variability. To update the information from time to time, recursive update approach is employed. Packet success rate computation is done using packet delivery ratio. The model used to update is taken from [13]. The estimation process is known as sequential estimation process using round trip time (RTT).

\section{Jamming-Aware Traffic Allocation}

This section explores the actual process of jamming aware traffic allocation using portfolio selection and also in-network inference correlation among variables. The traffic allocation and multiple path source routing takes place in presence of jammer. There are certain constraints to be considered. They include link capacity constraints, traffic flow reduction and source data rate constraints. Naturally the packet delivery ratio gets deteriorated when there is presence of jammer in the network. While making traffic allocation, on each path constraints pertaining to capacity are applied. In order to compensate randomness, redidual packet success rate concept is used depending on the capacity constraints [6]. For allocating traffic optimally, the theory of portfolio selection is employed [8] and [12]. Portfolio selection considered for real world financial investments decisions is presented in table 1 .

\begin{tabular}{|l|l|}
\hline \multicolumn{1}{|c|}{ Portfolio Selection } & \multicolumn{1}{|c|}{ Traffic Allocation } \\
\hline Funds to be invested & Source data rate \\
Financial lassets & Routing paths \\
Expected Assetreturn & Expected Packetsuccess rate \\
Investment portfolio & Traffic allocation \\
Portfolio return & Meanthroughput \\
Portfoliorisk & Estimation variance \\
\hline
\end{tabular}

Table 1 - Traffic Allocation and Portfolio Selection Comparison 
As seen in table1, there are portfolios in the real world mapped to the traffic allocation and the results thereof. The portfolio shows various investment options and their advantages and risks. This is compared with the advantages and risks of traffic allocation decisions in the network. For instance investment portfolio is compared with traffic allocation; return of portfolio is compared with mean throughput in the network while the portfolio risk is compared with estimation variance.

\section{Evaluation Of Emperical Results}

NS2 is used to simulate the network where source node allocates traffic to multiple paths in order to send it to destination. With respect to optimal traffic allocation, various techniques are used and the jamming impact is evaluated. The main focus is on the jamming statistics and portfolio selection besides consideration for in-network inference of correlation among the variables. Many techniques pertaining to traffic allocation are considered. There are two cases particularly. Ignoring the jamming is the first case. In case 2, presence of jammer is expected and maximum throughput is desired. Jamming aware optimization is to be carried out while allocating traffic to multiple paths. The source node is empowered to make use of the portfolio selection in order to achieve maximum throughput. The case 3 is to consider parameters pertaining to uncertainty in order to balance the mean throughput of the network. Its helps in continuous optimization.

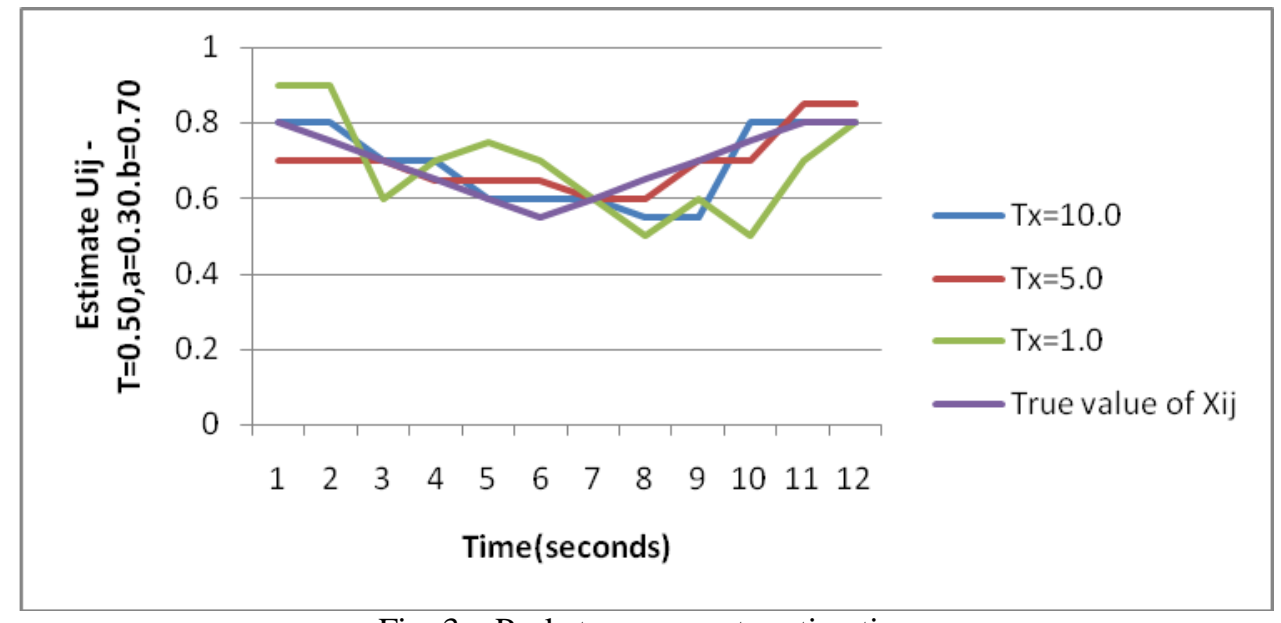

Fig. 3 - Packet success rate estimation

As seen in fig. 3, the $\mathrm{X}$ axis shows time in seconds while the $\mathrm{Y}$ axis shows the success rate estimation. These Details are used by source node while making routing decisions.

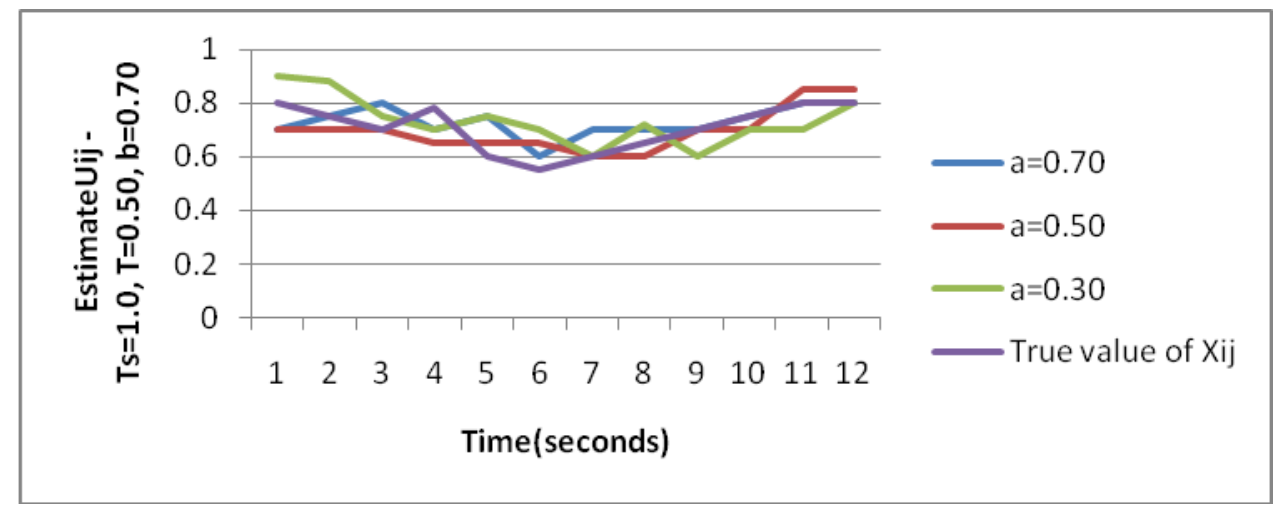

Fig. 4 -EWMA coefficients

As shown in fig. 4, the $\mathrm{X}$ axis shows time in seconds while the $\mathrm{Y}$ axis shows the success rate estimation details. 


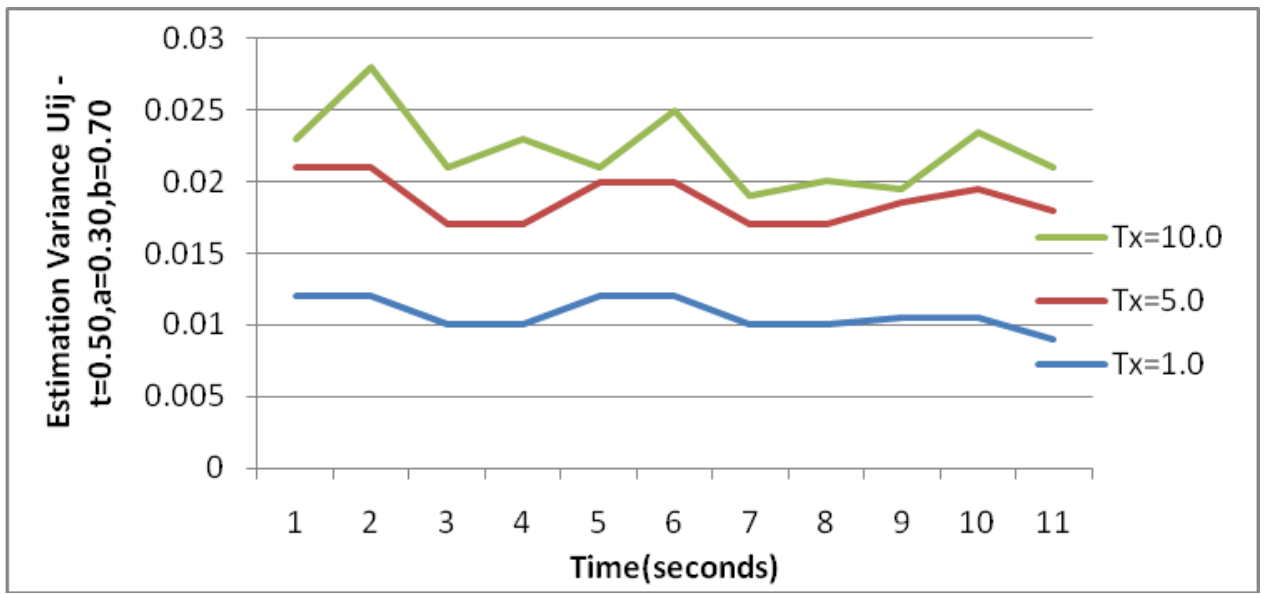

Fig. 5 - Packet success rate with variance

As seen in fig. 5, the $\mathrm{X}$ axis shows the time in seconds while the $\mathrm{Y}$ axis shows the success rate variance.

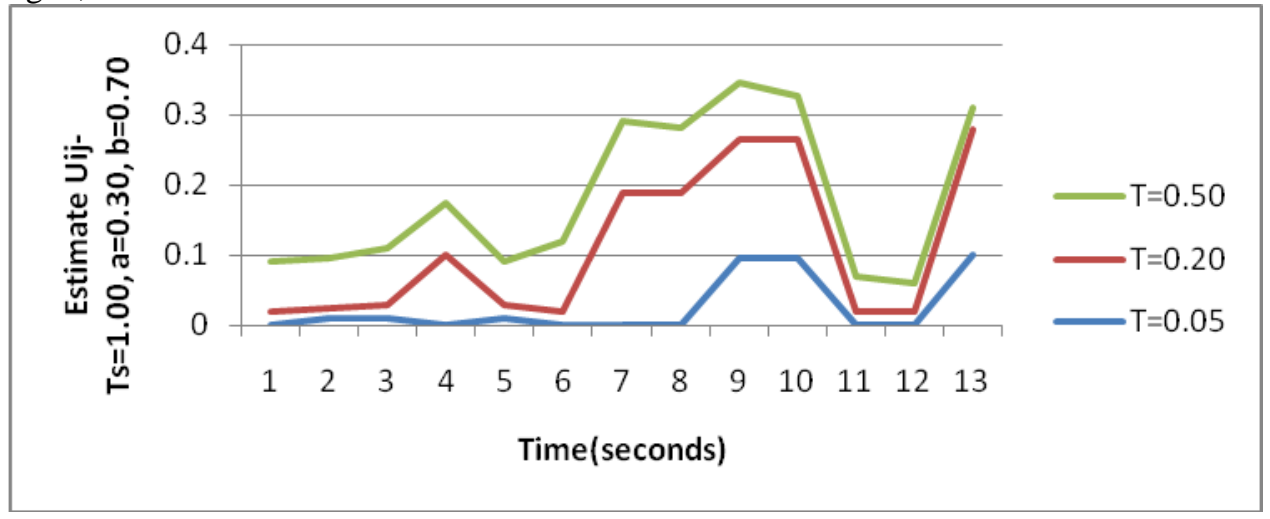

Fig. 6 - Estimation of Update Period

As seen in fig. 6, the update period estimate is shown in $\mathrm{Y}$ axis while the time in seconds is shown in $\mathrm{X}$ axis.

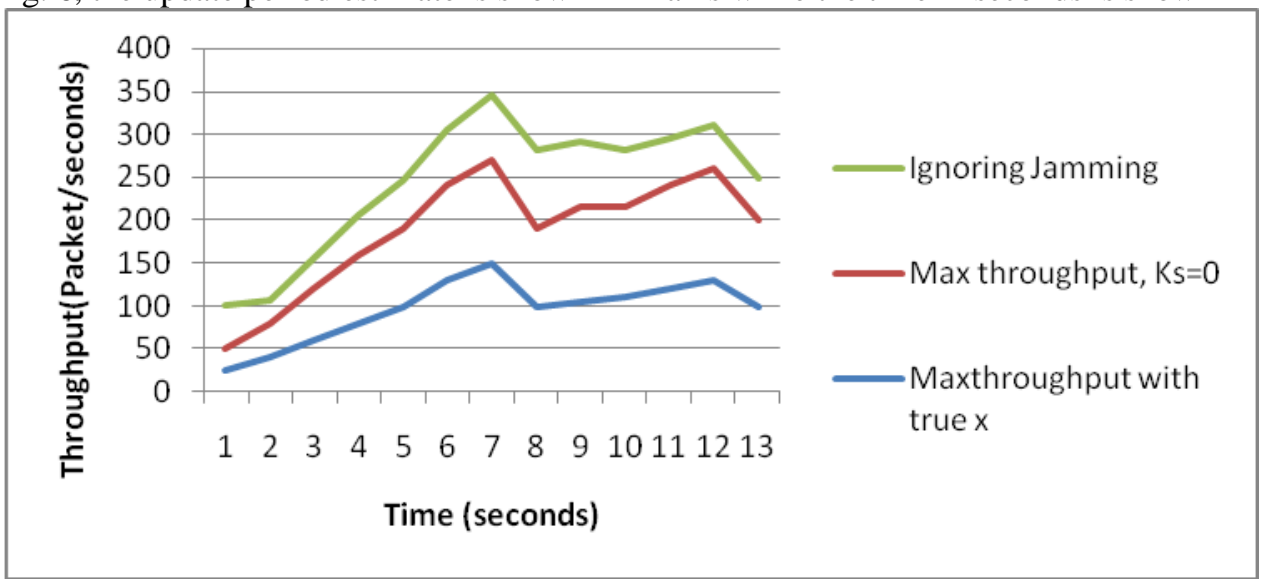

Fig. 7 -EWMA coefficients

As seen in fig. 7, the $\mathrm{X}$ axis shows time in seconds while the $\mathrm{Y}$ axis shows packets per second or throughput. 


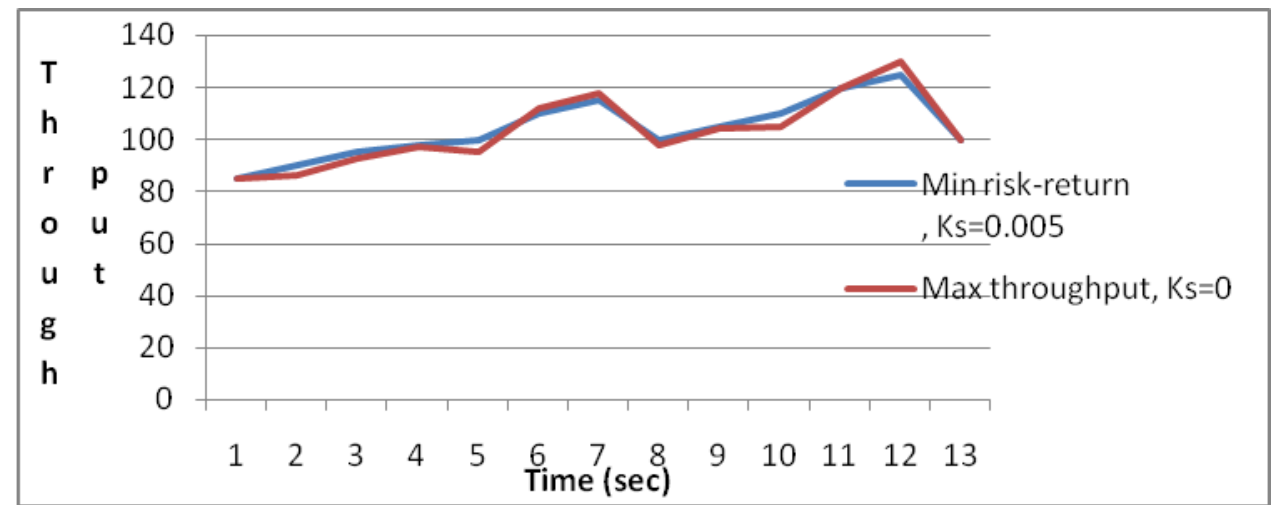

Fig. 8 - Simulated throughput

As shown in fig. 8, the $\mathrm{X}$ axis shows time in seconds while the $\mathrm{Y}$ axis shows the packets per second or throughput.

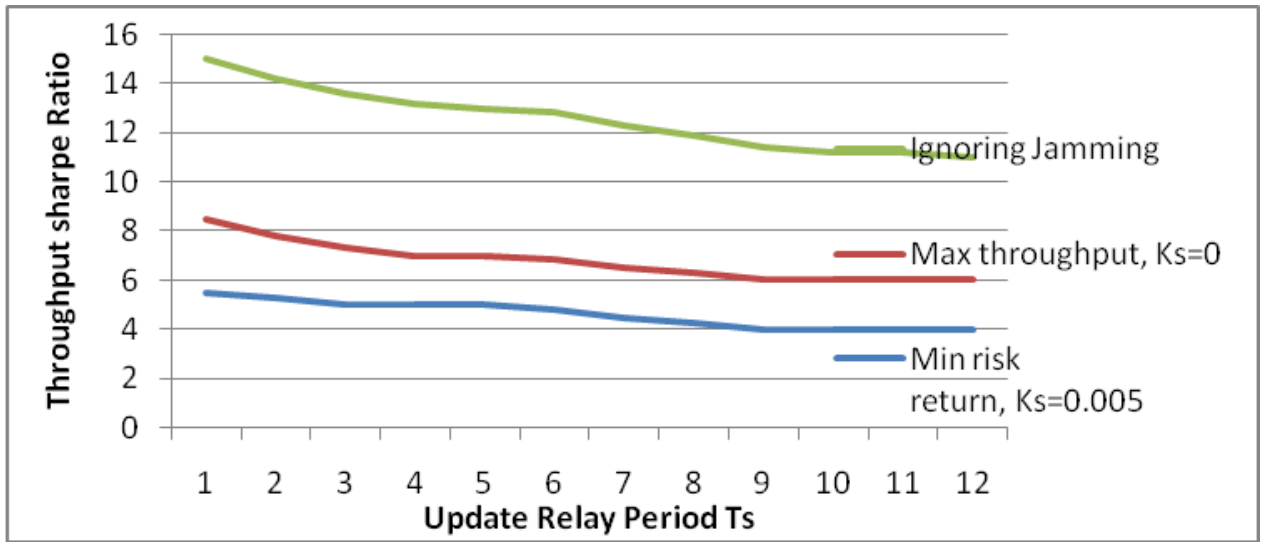

Fig. 9 - Updated relay and throughput

As shown in fig. 9, the $\mathrm{X}$ axis shows time in seconds while the $\mathrm{Y}$ axis shows the packets per second or throughput.

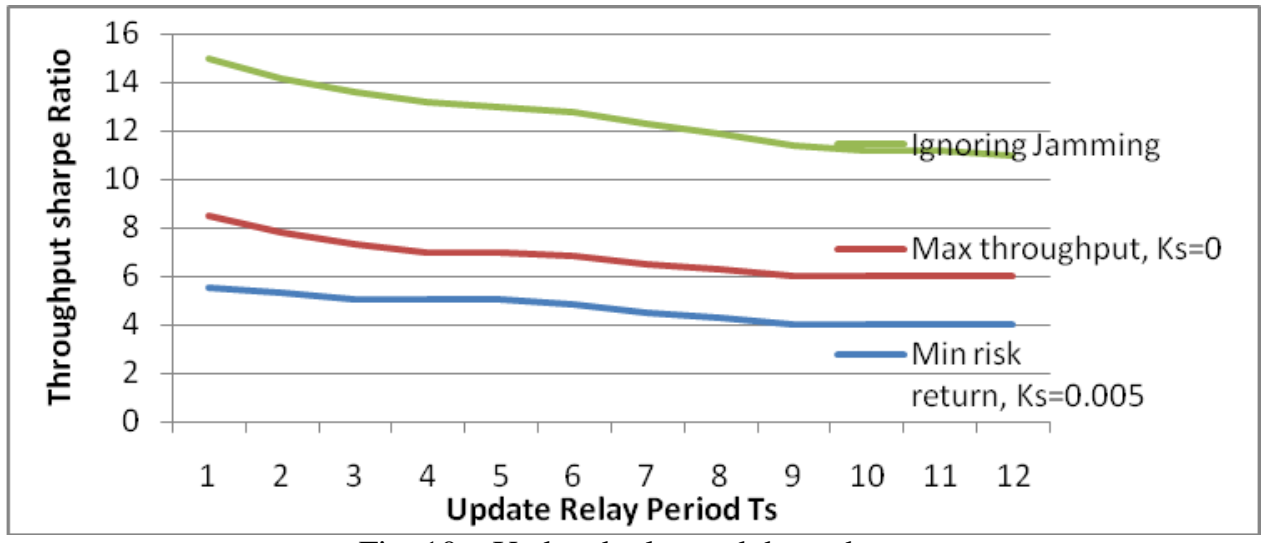

Fig. 10 - Updated relay and throughput

As seen in fig. 10, the $\mathrm{X}$ axis shows time in seconds while the $\mathrm{Y}$ axis shows throughput.

\section{Conclusion}

This paper provides implementation details and results of jamming-aware traffic allocation simulated in NS2. The traffic allocation and portfolio selection procedures implemented are based on [1]. It also relaxes the assumption considered in [1] with respect to in-network inference of correlations among related variables. In this implementation the source node allocates traffic to the available paths based on the awareness of jamming details at nodes. While using the methods of [1] this paper explores the statistics pertaining to in-network inference while implementing jamming-aware traffic allocation in multiple-path source routing networks. The empirical results revealed that the jamming aware traffic allocation methods with portfolio selection theory 
besides considering in-network inference resulted in network utility maximization and increase the throughput in presence of jammers.

\section{References}

[1] Patrick Tague, Sidharth Nabar, James A. Ritcey, and Radha Poovendran, "Jamming-Aware Traffic Allocation for Multiple-Path Routing Using Portfolio Selection”, IEEE/ACM TRANSACTIONS ON NETWORKING, VOL. 19, NO. 1, FEB 2011.

[2] R. Anderson, Security Engineering: A Guide to Building Dependable Distributed Systems. JohnWiley\&Sons, Inc.,2001.

[3] J. Bellardo and S. Savage, "802.11 denial-of-service attacks: Real vulnerabilities and practical solutions," in Proc. USENIX Security Symposium, Washington, DC, Aug. 2003, pp. 15-28.

[4] D. J. Thuente and M. Acharya, "Intelligent jamming in wireless networks with applications to $802.11 \mathrm{~b}$ and other networks," in Proc. $25^{\text {th }}$ IEEE Communications Society Military Communications Conference (MILCOM'06), Washington, DC, Oct. 2006 , pp. 1-7.

[5] G. Lin and G. Noubir, "On link layer denial of service in data wireless LANs," Wireless Communications and Mobile Computing, vol. 5, no. 3, pp. 273-284, May 2005.

[6] W. Xu, K. Ma, W. Trappe, and Y. Zhang, “Jamming sensor networks: Attack and defense strategies," IEEE Network, vol. 20, no. 3, pp. 41-47, May/Jun. 2006.

[7] R. Leung, J. Liu, E. Poon, A.-L. C. Chan, and B. Li, "MP-DSR: A QoSaware multi-path dynamic source routing protocol for wireless ad-hoc networks," in Proc. 26th Annual IEEE Conference on LocalComputer Networks (LCN'01), Tampa, FL, USA, Nov. 2001, pp. 132-141.

[8] H. Markowitz, "Portfolio selection," The Journal of Finance, vol. 7, no. 1, pp. 77-92, Mar. 1952.

[9] E. M. Sozer, M. Stojanovic, and J. G. Proakis, "Underwater acoustic networks," IEEE Journal of Oceanic Engineering, vol. 25, no. 1, pp. 72-83, Jan. 2000.

[10] A. D. Wood and J. A. Stankovic, "Denial of service in sensor networks," IEEE Computer, vol. 35, no. 10, pp. 54-62, Oct. 2002.

[11] E. M. Royer and C. E. Perkins, "Ad hoc on-demand distance vector routing," in Proc. 2nd IEEE Workshop on mobile Computing Systems and Applications (WMCSA'99), New Orleans, LA, USA, Feb. 1999, pp. 90-100.

[12] S. Boyd and L. Vandenberghe, Convex Optimization. Cambridge,2004.

[13] S. W. Roberts, "Control chart tests based on geometric moving averages," Technometrics, vol. 42, no. 1, pp. 97-101, Feb. 2000. 